\title{
A new family of almost identities
}

Gérard Maze and Lorenz Minder

Gérard Maze received his Ph.D. from the University of Notre Dame in 2002. He currently holds a postdoctoral position at the University of Zurich. His research interests lie in the field of Applied Algebra.

Lorenz Minder is currently a Ph.D. student at the École Polytechnique Fédérale de Lausanne (EPFL). His research interests cover cryptography and coding theory.

\section{Introduction}

It is well-known that a class of "almost integers" can be found using the theory of modular functions, and a few spectacular examples are given by Ramanujan [5]. They can be generated using some amazing properties of the $j$-function. Some of the numbers which are close approximations of integers are $\exp (\pi \sqrt{163})$ (sometimes known as Ramanujan's constant $), \exp (\pi \sqrt{37})$ and $\exp (\pi \sqrt{58})$. These irrationals come close to an integer as follows:

$$
\begin{aligned}
\exp (\pi \sqrt{37}) & =199148648-0.219 \ldots \cdot 10^{-4} \\
\exp (\pi \sqrt{58}) & =24591257752-0.177 \ldots \cdot 10^{-6} \\
\exp (\pi \sqrt{163}) & =262537412640768744-0.749 \ldots \cdot 10^{-12} .
\end{aligned}
$$

Another surprising result comes from the average length of a segment in an isosceles right triangle with catheti of unit length. If $l$ is this average length, then

$$
l=\frac{1}{30}\left(2+4 \sqrt{2}+(4+\sqrt{2}) \sinh ^{-1}(1)\right)=0.4142933026 \ldots=(\sqrt{2}-1)-0.8 \ldots \cdot 10^{-4} .
$$

„Almost identities“ sind mathematische Ausdrücke, die gegen „schöne“ Zahlen zu konvergieren scheinen, stattdessen aber - mit oft schier unglaublicher Präzision - danebentreffen. Verblüffende almost identities sind faszinierend. Noch faszinierender ist aber meist die Frage, die almost identities unweigerlich aufwerfen, nämlich: Ist es Zufall oder gibt es einen tieferen Grund? In dieser Arbeit stellen die Autoren eine Familie von almost identities vor und geben anschliessend eine Analyse, die das Phänomen in diesen Fällen erklärt. 
Such astonishing non-equalities are usually called almost identities or non-identities. Many examples of such unexpected behaviour are known [6]. The four examples above are however different in essence: the first three come from a deep property of a complex mathematical object (the $j$-function) and the last has a good chance of being a genuine arithmetical coincidence.

A natural question that comes to mind in presence of such a non-identity is therefore whether or not the phenomenon is purely coincidental, or comes from a more subtle process. For instance, in the equation

$$
e^{\pi}-\pi=19.999099979 \ldots,
$$

it is not clear at all whether the almost identity pops up from a deep connection between $e$ and $\pi$ or just because the expression happens to be close to 20 .

Recently, J.M. Borwein and P.B. Borwein discovered several families of almost identities [2], leading to a systematic study of such phenomena. These were based on mathematical concepts that lead to clear explanations. Among the non-identities studied by these authors, let us mention the following striking example:

$$
\sum_{k=-\infty}^{\infty} \frac{1}{10^{(k / 100)^{2}}} \cong 100 \sqrt{\frac{\pi}{\ln (10)}}
$$

correct to at least 18,000 digits. In this situation, the almost identity is not a coincidence. From the same viewpoint, let us mention as well the sequence

$$
h_{n}=\frac{n !}{2(\ln (2))^{n+1}},
$$

for $1 \leqslant n \leqslant 17$, discovered by $\mathrm{D}$. Hickerson. These numbers are close to integers due to the fact that the above quotient is the dominant term in an infinite series whose sum is the number of possible outcomes of a race between $n$ people (where ties are allowed). See [6] for the exact expression of these numbers. Here, once again, no coincidence.

While we were studying the function

$$
\left.\left.f(x)=\sum_{k=1}^{\infty} \frac{1}{1+2^{k} x}, \quad x \in\right] 0,1\right]
$$

that appears in the analysis of the complexity of the binary ged algorithm, we came to find a new family of almost identities. Let us define the real numbers $u_{n}$ as follows:

$$
u_{n}:=\ln (2) \cdot \sum_{k=-\infty}^{\infty} \frac{1}{\left(2^{k / 2}+2^{-k / 2}\right)^{n}}, \quad n \in \mathbb{N} \backslash\{0\} .
$$

The following equalities show the very strange behaviour of the almost identities generated 
by the sequence $\left\{u_{n}\right\}$ :

$$
\begin{gathered}
u_{1}=\pi+0.53 \ldots \cdot 10^{-11} \\
u_{2}=1+0.48 \ldots \cdot 10^{-10} \\
u_{3}=\frac{\pi}{2^{3}}+0.22 \ldots \cdot 10^{-9} \\
u_{4}=\frac{1}{6}+0.67 \ldots \cdot 10^{-9} \\
u_{5}=\frac{3 \pi}{2^{7}}+0.15 \ldots \cdot 10^{-8} \\
u_{6}=\frac{1}{30}+0.29 \ldots \cdot 10^{-8} \\
\vdots \vdots
\end{gathered}
$$

This article presents an explanation of this phenomenon. We study the cases with $n=1$ and $n=2$ by using the theory of Mellin transforms and leave the remaining cases together with the recurrence relation (1.1) below to the extended version of this article [4]. We also present a generalization of the phenomenon, leading to, e.g., the almost identity

$$
\ln (4) \cdot \sum_{k=-\infty}^{\infty} \frac{1}{2^{-k}+2^{k}}=\pi+0.82 \ldots \cdot 10^{-5} \text {. }
$$

The proof of the recurrence relation

$$
u_{n}=\left(\frac{1}{4} \cdot \frac{n-2}{n-1}\right) u_{n-2}+r_{n}
$$

is not given in this article, but it can be found in the extended version of this work [4], where also the explicit values of $r_{n}$ satisfying $0<r_{n} \leqslant r_{10}=0.7227399 \ldots \cdot 10^{-8}$, $\forall n \in \mathbb{N}$, are described.

In this article, we will use the notation $f(x) \sim_{a} g(x)$ for equivalent functions in a neighbourhood of $a$ and $\log _{2} x$ for the logarithm in base 2 of $x$. Also, the set $\mathbb{N}$ is considered to contain the integer 0 in the sequel.

\section{Preliminaries}

The first two cases on our list are

$$
u_{1}=\ln (2) \cdot \sum_{k=-\infty}^{\infty} \frac{1}{2^{-k / 2}+2^{k / 2}}=\pi+0.53 \ldots \cdot 10^{-11}
$$

and

$$
u_{2}=\ln (2) \cdot \sum_{k=-\infty}^{\infty} \frac{1}{\left(2^{-k / 2}+2^{k / 2}\right)^{2}}=1+0.48 \ldots \cdot 10^{-10} \text {. }
$$


Let us define the complex functions $g_{1}$ and $g_{2}$ as

$$
g_{1}(x)=-2 \cdot(\arctan (\sqrt{x})-\pi / 2) \text { and } g_{2}(x)=\frac{1}{1+x}, \quad \Re x>0,
$$

as well as the functions $G_{1}$ and $G_{2}$ defined as

$$
G_{n}(x)=\sum_{k=1}^{\infty} g_{n}\left(2^{k} x\right), \quad \Re x>0, \quad n=1,2 .
$$

The convergence of $G_{1}$ is justified by the fact that in a neighbourhood of $+\infty$ we have

$$
\arctan t-\pi / 2=-\int_{t}^{\infty} \frac{1}{1+v^{2}} d v=-\int_{t}^{\infty}\left(\frac{1}{v^{2}}-\frac{1}{v^{4}}+\frac{1}{v^{6}}+\cdots\right) d v=O(1 / t) .
$$

The following equalities are justified because $G_{1}$ and $G_{2}$ converge uniformly on compact subsets of their domains, and therefore, the derivative can be interchanged with the sum. Therefore,

$$
\begin{aligned}
\left.\lim _{m \rightarrow \infty} \frac{d}{d u}\left[G_{n}\left(2^{-u}\right)\right]\right|_{u=m} & =\left.\lim _{m \rightarrow \infty} \sum_{k=1}^{\infty} \frac{d}{d u}\left[g_{n}\left(2^{k-u}\right)\right]\right|_{u=m} \\
& =\lim _{m \rightarrow \infty} \ln (2) \cdot \sum_{k=1}^{\infty} \frac{\left(2^{(k-m)}\right)^{n / 2}}{\left(1+2^{(k-m)}\right)^{n}} \\
& =\lim _{m \rightarrow \infty} \ln (2) \cdot \sum_{k=1}^{\infty} \frac{1}{\left(2^{-(k-m) / 2}+2^{(k-m) / 2}\right)^{n}} \\
& =u_{n},
\end{aligned}
$$

where the limit is understood with $m \in \mathbb{N}$. The game plan is then to express the functions $G_{1}$ and $G_{2}$ in a completely different manner in order to compute these limits. The keystone of this process is the Mellin transform [3]. Recall that the Mellin transform of a locally Lebesgue integrable function $f(x)$ over $] 0, \infty[$ is the function

$$
f^{*}(s)=\int_{0}^{\infty} f(x) x^{s-1} d x
$$

The conditions $f(x) \sim_{0} O\left(x^{u}\right)$ and $f(x) \sim_{\infty} O\left(x^{v}\right)$, with $u>v$ guarantee that $f^{*}(s)$ exists in the strip $-u<\Re s<-v$. Mellin's inversion formula [3, p. 13] states that if $f$ is continuous and $c \in]-u,-v[$, then

$$
f(x)=\frac{1}{2 \pi i} \int_{c-i \infty}^{c+i \infty} f^{*}(s) x^{-s} d s
$$

and in a neighbourhood of 0 , we have

$$
f(x)=\sum_{\Re s_{l}<c} \operatorname{Res}\left(f^{*}(s) x^{-s}, s_{l}\right),
$$


where the summation is over the poles $s_{l}$ of the function $f^{*}(s) x^{-s}$ whose real part is strictly smaller than $c$.

Let $g(x)$ be a locally Lebesgue integrable function over ]0, $\infty\left[, f(x)=\sum_{k=1}^{\infty} g\left(2^{k} x\right)\right.$, and suppose that the convergence is uniform in $] 0, \infty[$. Then,

$$
\begin{aligned}
f^{*}(s) & =\int_{0}^{\infty} \sum_{k=1}^{\infty} g\left(2^{k} x\right) x^{s-1} d x \\
& =\sum_{k=1}^{\infty} \int_{0}^{\infty} g(y) y^{s-1} 2^{-k s} d y \\
& =\frac{g^{*}(s)}{2^{s}-1} .
\end{aligned}
$$

\section{The case $n=1$}

Proposition 1. For $x>0$, we have

$$
G_{1}(x)=-\frac{\pi}{2}-\pi \log _{2}(x)+\sqrt{x} S_{1}(x)-\sum_{k=1}^{\infty} \frac{\sin \left(2 k \pi \log _{2}(x)\right)}{k \cdot \cosh \left(2 k \pi^{2} / \ln (2)\right)},
$$

where $S_{1}(x)$ is a power series in $x$, which converges in $[0,1[$.

Proof. As announced earlier, the idea is to use Mellin transforms in a back and forth process to reveal another expression of $G_{1}$. Using (2.2), we can write

$$
G_{1}^{*}(s)=\frac{g_{1}^{*}(s)}{2^{s}-1} .
$$

In order to compute $g_{1}^{*}$, recall that in a neighbourhood of $+\infty$ we have $\arctan t-\pi / 2=$ $O(1 / t)$. So, we can perform an integration by parts, as long as $\Re s \in] 0,1 / 2[$ :

$$
\begin{aligned}
g_{1}^{*}(s) & =-2 \int_{0}^{\infty}(\arctan (\sqrt{x})-\pi / 2) x^{s-1} d x \\
& =-2 \cdot\left[\left.(\arctan (\sqrt{x})-\pi / 2) \cdot \frac{x^{s}}{s}\right|_{0} ^{\infty}-\frac{1}{2 s} \int_{0}^{\infty} \frac{x^{s-1 / 2}}{1+x} d x\right] \\
& =\frac{1}{s} \int_{0}^{\infty} \frac{x^{s-1 / 2}}{1+x} d x \\
& =\frac{\pi}{s \cos \pi s} .
\end{aligned}
$$

The last equality comes from the relation

$$
\int_{0}^{\infty} \frac{x^{s-1}}{1+x} d x=\frac{\pi}{\sin \pi s}
$$


Using Mellin's inversion formula with $c=1 / 4$ and (3.1), we get

$$
\begin{aligned}
G_{1}(x) & =\frac{1}{2 \pi i} \int_{1 / 4-i \infty}^{1 / 4+i \infty}\left(\frac{\pi}{s \cos \pi s}\right) \frac{x^{-s}}{2^{s}-1} d s \\
& =\sum_{\Re_{s_{l}<1 / 4}} \operatorname{Res}\left(\left(\frac{\pi}{s \cos \pi s}\right) \frac{x^{-s}}{2^{s}-1}, s_{l}\right) .
\end{aligned}
$$

The poles of the function $\left(\frac{\pi}{s \cos \pi s}\right) \frac{x^{-s}}{2^{s}-1}$ can be partitioned as follows:

i) $s=0$ is a pole of order two,

ii) the real simple poles $-1 / 2+k, k \in \mathbb{Z}$,

iii) the imaginary simple poles $2 k \pi i / \ln (2), k \in \mathbb{Z} \backslash\{0\}$.

The residues are then

$$
\begin{aligned}
-\pi \log _{2}(x)-\frac{\pi}{2} & \text { at } s=0, \\
-\frac{(-2)^{k+2}}{(1+2 k)\left(2^{k+1}-\sqrt{2}\right)} \sqrt{x} x^{k} & \text { at } s=-1 / 2-k, k \in \mathbb{N}, \\
\frac{1}{2 i} \cdot \frac{\exp \left(-2 k \pi i \log _{2}(x)\right)}{k \cdot \cosh \left(2 k \pi^{2} / \ln (2)\right)} & \text { at } s=2 k \pi i / \ln (2), k \in \mathbb{Z} \backslash\{0\},
\end{aligned}
$$

and the above sum becomes

$$
\begin{aligned}
G_{1}(x)=-\frac{\pi}{2}-\pi \log _{2}(x) & +\sum_{k=0}^{\infty} \frac{(-2)^{k+2}}{(1+2 k)\left(-2^{k+1}+\sqrt{2}\right)} \sqrt{x} x^{k} \\
& -\sum_{k=1}^{\infty} \frac{\sin \left(2 k \pi \log _{2}(x)\right)}{k \cdot \cosh \left(2 k \pi^{2} / \ln (2)\right)}
\end{aligned}
$$

which proves the proposition.

Corollary 2. $u_{1}=\pi+\sum_{k=1}^{\infty} \frac{2 \pi}{\cosh \left(2 k \pi^{2} / \ln (2)\right)}$.

Proof. Based on (2.1), we have

$$
\begin{aligned}
u_{1} & =\left.\lim _{m \rightarrow \infty} \frac{d}{d u}\left[G_{1}\left(2^{-u}\right)\right]\right|_{u=m} \\
& =\pi+\lim _{u \rightarrow \infty}\left[e^{-u / 2} S_{1}\left(e^{-u}\right)\right]^{\prime}+\sum_{k=1}^{\infty} \frac{2 \pi}{\cosh \left(2 k \pi^{2} / \ln (2)\right)}
\end{aligned}
$$

and the last limit being equal to zero, the corollary is proven. 
The case $n=1$ is then settled since the sum on the right-hand side of the equality of Corollary 2 is in fact small:

$$
u_{1}-\pi=\sum_{k=1}^{\infty} \frac{2 \pi}{\cosh \left(2 k \pi^{2} / \ln (2)\right)}=0.538914478 \ldots \cdot 10^{-11}
$$

\section{The case $n=2$}

We can compute $u_{2}$ in a similar way as we computed $u_{1}$. Note that once $u_{1}$ and $u_{2}$ are computed, the recurrence relation (1.1) gives the complete sequence $\left\{u_{i}\right\}$.

Proposition 3. For $x>0$, we have

$$
G_{2}(x)=-\frac{1}{2}-\log _{2}(x)+S_{2}(x)-\frac{2 \pi}{\ln (2)} \sum_{k=1}^{\infty} \frac{\sin \left(2 k \pi \log _{2}(x)\right)}{\sinh \left(2 k \pi^{2} / \ln (2)\right)},
$$

where $S_{2}(x)$ is a power series in $x$, converging in $\left[0,1\left[\right.\right.$ such that $S_{2}(x)=0$.

Proof. The proof follows the same lines as in the first case. First,

$$
g_{2}^{*}(s)=\int_{0}^{\infty} \frac{x^{s-1}}{1+x} d x=\frac{\pi}{\sin \pi s},
$$

and thus, once again based on (2.2) and (3.1), we have

$$
\begin{aligned}
G_{2}(x) & =\int_{1 / 2-i \infty}^{1 / 2+i \infty} G_{2}^{*}(s) x^{-s} d s \\
& =\int_{1 / 2-i \infty}^{1 / 2+i \infty}\left(\frac{\pi}{\sin \pi s}\right) \frac{x^{-s}}{2^{s}-1} d s \\
& =\sum_{i_{s}<1 / 2} \operatorname{Res}\left(\left(\frac{\pi}{\sin \pi s}\right) \frac{x^{-s}}{2^{s}-1}, s_{l}\right) .
\end{aligned}
$$

The poles of the function can be partitioned as follows:

i) $s=0$ is a pole of order two,

ii) the real simple poles $k, k \in \mathbb{Z} \backslash\{0\}$,

iii) the imaginary simple poles $2 k \pi i / \ln (2), k \in \mathbb{Z} \backslash\{0\}$.

The residues are then

$$
\begin{aligned}
-\log _{2}(x)-\frac{1}{2} & \text { at } s=0, \\
-\frac{(-2)^{k}}{2^{k}-1} x^{k} & \text { at } s=-k, k=1,2,3, \ldots, \\
\frac{\pi}{i} \cdot \frac{\exp \left(-2 k \pi i \log _{2}(x)\right)}{\ln (2) \cdot \sinh \left(2 k \pi^{2} / \ln (2)\right)} & \text { at } s=2 k \pi i / \ln (2), k \in \mathbb{Z} \backslash\{0\} .
\end{aligned}
$$


The new expression of $G_{2}$ is therefore

$$
G_{2}(x)=-\frac{1}{2}-\log _{2}(x)-\sum_{k=1}^{\infty} \frac{(-2)^{k}}{2^{k}-1} x^{k}-\frac{2 \pi}{\ln (2)} \sum_{k=1}^{\infty} \frac{\sin \left(2 k \pi \log _{2}(x)\right)}{\sinh \left(2 k \pi^{2} / \ln (2)\right)}
$$

This concludes the proof.

Corollary 4. $u_{2}=1+\frac{2 \pi}{\ln (2)} \sum_{k=1}^{\infty} \frac{2 k \pi}{\sinh \left(2 k \pi^{2} / \ln (2)\right)}$.

Proof. We use the same trick as in Corollary 2:

$$
\begin{aligned}
u_{2} & =\left.\lim _{m \rightarrow \infty} \frac{d}{d u}\left[G_{2}\left(2^{-u}\right)\right]\right|_{u=m} \\
& =1+\lim _{u \rightarrow \infty}\left[S_{2}\left(e^{-u}\right)\right]^{\prime}+\frac{2 \pi}{\ln (2)} \sum_{k=1}^{\infty} \frac{2 k \pi}{\sinh \left(2 k \pi^{2} / \ln (2)\right)}
\end{aligned}
$$

and the limit being equal to zero, the corollary is proven.

Once again, this shows why the number $u_{2}$ is almost an integer. Indeed, the sum on the right-hand side is fairly small:

$$
u_{2}-1=\frac{2 \pi}{\ln (2)} \sum_{k=1}^{\infty} \frac{2 k \pi}{\sinh \left(2 k \pi^{2} / \ln (2)\right)}=0.4885108992 \ldots \cdot 10^{-10}
$$

\section{Final remarks}

Having found the roots of the mystery related to the non-equalities $u_{1} \neq \pi$ and $u_{2} \neq 1$, let us mention that the same tools used so far lead to a complete understanding of the nonequalities concerning $u_{3}, u_{4}, \ldots$ and why they are so close to "good arithmetic numbers". The interested reader can find the details in the extended version of this article [4].

We end this article with the following remark: Our arguments do not depend on the presence of 2 in the denominator of

$$
\frac{1}{\left(2^{-k / 2}+2^{k / 2}\right)^{n}}
$$

One could argue that any sum of the type

$$
\ln (m) \cdot \sum_{k=-\infty}^{\infty} \frac{1}{\left(m^{-k / 2}+m^{k / 2}\right)^{n}}
$$


has the potential to lie close to $\mathbb{Q}$ or $\pi \mathbb{Q}$ depending on the parity of $n$. As a matter of fact, we have, for example,

$$
\begin{array}{r}
\ln (4) \cdot \sum_{k=-\infty}^{\infty} \frac{1}{2^{-k}+2^{k}}=\pi+0.82 \ldots \cdot 10^{-5}, \\
\ln (9) \cdot \sum_{k=-\infty}^{\infty} \frac{1}{3^{-k}+3^{k}}=\pi+0.15 \ldots \cdot 10^{-2}, \\
\ln (4) \cdot \sum_{k=-\infty}^{\infty} \frac{1}{\left(2^{-k}+2^{k}\right)^{2}}=1+0.37 \ldots \cdot 10^{-4} .
\end{array}
$$

In fact, it can be proven that the choice $m=2$ is the best one can do in order to maximize the resemblance with elements in $\mathbb{Q} \cup \pi \mathbb{Q}$, see [4].

\section{References}

[1] Andrews, G.E.; Askey, R.; Roy, R.: Special functions. Encyclopedia of Mathematics and its Applications, 71, Cambridge University Press, 1999.

[2] Borwein, J.M.; Borwein, P.B.: Strange Series and High Precision Fraud. Amer. Math. Monthly 99 (1992), $622-640$.

[3] Flajolet, P.; Gourdon, X.; Dumas, P.: Mellin transforms and asymptotics: harmonic sums. Theoret. Comput. Sci. (Special volume on mathematical analysis of algorithms) 144 (1995), 3-58.

[4] Maze, G.; Minder, L.: A New Family of Almost Identities (extended version). http://www.arxiv.org/abs/math.GM/0409014.

[5] Ramanujan, S.: Modular Equations and Approximations to $\pi$. Quart. J. Pure Appl. Math. 45 (1914/15), $350-372$.

[6] Weisstein, E.W.: Almost Integer. From MathWorld - A Wolfram Web Resource, at http://mathworld.wolfram.com/AlmostInteger.html.

\section{Gérard Maze}

Mathematics Institute

University of Zurich

CH-8057 Zurich, Switzerland

e-mail: gmaze@math.unizh.ch

Lorenz Minder

Laboratoire de Mathématiques Algorithmiques

École Polytechnique Fédérale de Lausanne

CH-1015 Lausanne, Switzerland

e-mail: lorenz.minder@epfl.ch 